\title{
Measuring resilience with the Connor-Davidson Resilience Scale (CD-RISC): which version to choose?
}

\author{
Heleen Kuiper ${ }^{1,2} \cdot$ Christel C. M. van Leeuwen $^{1,3} \cdot$ Janneke M. Stolwijk-Swüste ${ }^{1,3} \cdot$ Marcel W. M. Post $^{1,2}$
}

Received: 23 October 2018 / Revised: 20 December 2018 / Accepted: 20 December 2018 / Published online: 22 January 2019

(c) International Spinal Cord Society 2019

\begin{abstract}
Study design Cross-sectional psychometric study.

Objectives To compare psychometric properties of the Connor-Davidson Resilience Scale (CD-RISC) with 25, 10, and 2 items, and to assess the agreement between these versions in individuals with spinal cord injury (SCI).

Setting Standard psychological screening at a Dutch rehabilitation centre during the first 2 weeks of inpatient rehabilitation. Methods Anonymous data from the psychological screening were analysed. CD-RISC outcomes were checked for floor and ceiling effects. Internal consistency was assessed by calculating Cronbach's $\alpha$. Convergent validity was assessed by Spearman's correlation between resilience and anxiety, depression, passive coping, and life satisfaction. Agreement between CD-RISC versions was examined by calculating intraclass correlation coefficients (ICCs), corresponding 95\% confidence intervals (CIs), and Bland-Altman plots.

Results Total CD-RISC scores were only skewed on the CD-RISC $2(-1.12)$. There were no floor and ceiling effects. Internal consistency of the 25-, 10-, and 2-item scales was good to moderate $(0.90,0.86$, and 0.66, respectively). Good convergent validity was shown only for the CD-RISC 10. Agreement was highest between the CD-RISC 25 and CD-RISC 10 with an ICC of 0.90 with $95 \%$ CI from 0.85 to 0.94 .

Conclusions Out of the three CD-RISC versions, the CD-RISC 10 showed the best combination of reliability, validity, and practicality. Therefore, this version is advised as measure of resilience in individuals with SCI in a rehabilitation setting. Measurement of resilience could be part of a psychological screening to identify individuals at risk to develop psychological problems after SCI.
\end{abstract}

\section{Introduction}

Resilience embodies the personal characteristics that enable one to thrive in the face of adversities, such as a spinal cord injury (SCI) [1]. Individuals with SCI are at risk for poor adjustment to this disabling condition [2,3]. Support of mental recovery after the onset of SCI is, therefore, an

Marcel W. M. Post

m.post@dehoogstraat.nl

1 Centre of Excellence for Rehabilitation Medicine, Brain Centre Rudolf Magnus, University Medical Centre Utrecht, Utrecht University and De Hoogstraat Rehabilitation, Utrecht, The Netherlands

2 University of Groningen, University Medical Centre Groningen, Centre for Rehabilitation, Groningen, The Netherlands

3 Department of Spinal Cord Injury and Orthopedics, De Hoogstraaat Rehabilitation, Utrecht, The Netherlands important part of the multidisciplinary treatment during initial rehabilitation [4]. Focussing on strengths, such as resilience, instead of focussing on a negative mental state, contributes to the prevention of pathology and helps to maintain and even improve physical and psychological well-being [5]. Previous studies have shown the potential of improving resilience by practicing positive psychology interventions in individuals with SCI [6]. The identification of individuals with low levels of resilience early after injury is, therefore, important in the rehabilitation of individuals with SCI.

A methodological review describes 15 different resilience measurement scales [7], of which the ConnorDavidson Resilience Scale (CD-RISC) 25 is one of the most regularly used scales with the best psychometric properties [7]. Two abbreviated versions of the CD-RISC 25 have been published. The 10-item version is proposed in response to a number of problems with the 25 -item version in exploratory factor analysis [8]. Around the same period, 
the 2-item version is introduced with the purpose of decreasing administration time and increasing usage [9].

A literature search has revealed that the CD-RISC has already been used among individuals with SCI [10-20]. In 2 of the 11 studies, psychometric properties of the CD-RISC are examined, but both in multi-diagnostic samples. In one study, high internal consistency reliability is reported, with Cronbach $\alpha 0.93$ for the 25 -item version, 0.88 for the 10 item version, and 0.76 for the 2 -item version among individuals with severe accidental injuries $(N=115)$, including 30 participants with paraplegia in a rehabilitation setting [10]. Based on good psychometric properties and practical use, the authors support the application of the 10-item version compared to the $25-$ and 2 -item versions [10]. The other study also found the psychometric properties of the 10 -item version to be adequate among adults with disabilities, including SCI, in a community setting [20].

Only 4 out of the 11 studies include exclusively individuals with SCI in inpatient rehabilitation, and none of these 4 compare the psychometric properties of the three versions of the CD-RISC. Since there is potential to assess resilience as part of the multidisciplinary inpatient rehabilitation treatment, it is important to apply a practical, reliable, and valid measure to rate resilience in individuals with a recently acquired SCI.

Therefore, the research questions of this study are: (1) Do the CD-RISC 25, CD-RISC 10, and CD-RISC 2 show equal reliability and validity in individuals with a recently acquired SCI in inpatient rehabilitation? (2) What is the agreement between the CD-RISC 25, CD-RISC 10, and CD-RISC 2 in this population? We hypothesised that: (1) all three versions would show good psychometric properties, meaning they show no floor or ceiling effects, show strong correlations with scores on measures of anxiety, depression, passive coping, and life satisfaction, and weak correlations with cause of SCI, gender, level and completeness of the SCI, and age; and (2) all three versions would show high agreement with each other, and that the 10-item and the 2-item versions are in agreement with the 25 -item version.

\section{Methods}

The study consisted of a cross-sectional psychometric study of prospectively collected data.

\section{Participants and procedure}

A brief psychological screening is part of the standard psychological intake with every individual with a recent $\mathrm{SCI}$ in our rehabilitation centre. Anonymous data out of the psychological screenings that were performed between March 2016 until November 2017 were retrieved from medical files by the treating psychologist $(\mathrm{CCMvL})$. Inclusion criteria were: (1) inpatient rehabilitation because of a recently acquired SCI or cauda equina syndrome, (2) minimum age of 18 years at the time of the psychological screening, and (3) completed at least the items covering the CD-RISC 10 and CD-RISC 2. The CD-RISC 10 and CDRISC 2 scores were extracted from the completed original version.

\section{Measures}

Resilience was measured with the CD-RISC 25 . The original CD-RISC comprises 25 statements on how one has felt over the past month. The response scale has a 5-point range: 0 (not true at all), 1 (rarely true), 2 (sometimes true), 3 (often true), and 4 (true nearly all of the time). Scores are added up to a maximum score of 100 , meaning high resilience [1]. The shortened version CD-RISC 10 includes 10 items that scored best on salient loadings on the 'hardiness' and 'persistence' factors, with a maximum overall score of 40 [8]. The 2-item CD-RISC 2 includes only statement 1 ('Able to adapt to change') and statement 8 ('Tend to bounce back after illness or hardship') with a maximum score of 8 [9].

Anxiety and depression were measured with the Hospital Anxiety and Depression Scale (HADS). The HADS consists of 14 statements about the past week with a 4-point response scale (score $0-3$ ). These 14 statements are split up into 7 anxiety indicators and 7 depression indicators. For each scale, a maximum score of 21 can be obtained, indicating a high probability of anxiety/ depression [21]. Scores of 0-7 indicate 'non-(clinical) cases', 8-10 'possible/ doubtful cases', and $\geq 11$ 'clinical/ definite cases' [22]. The HADS has proven to be a valid and reliable measure of anxiety/ depressive feelings in individuals with SCI [23, 24].

Passive coping was measured by the passive coping subscale from the Utrechtse Coping List (UCL) [25]. This subscale comprises seven statements, describing reactions to problems. Individuals indicate whether these reactions are 'rarely or never', 'sometimes', 'often', or 'very often' applicable for them. The total score ranges from 7 to 28 and a high score indicates high use of passive coping strategies. Norm values classify the total score as being 'very high', 'high', 'average', 'low', or 'very low', compared with healthy control population [25]. The UCL is shown to be valid and has been used in individuals with SCI before [2527].

Life satisfaction was measured with the two Life Satisfaction questions (2LS). The first question asks about satisfaction with one's life in the present that can be answered on a 6-point scale, from 1 (very unsatisfying) up to 6 (very satisfying). The second question asks to compare 
life satisfaction in the present to the situation before the SCI on a 7-point scale, from 1 (much worse) up to 7 (much better). Answers on both questions were summed to calculate an overall score (range 2-13) of life satisfaction. This scale has found to be valid in individuals with SCI [28].

\section{Statistical analyses}

Statistical analyses were performed using SPSS, version 25 (IBM, Armonk NY). Floor and ceiling effects were considered to be present if $15 \%$ or more of the individuals achieved the lowest or highest possible scores on the CDRISC, respectively. Skewness between -1 and 1 was considered acceptable. Internal consistency was examined using Cronbach's $\alpha$, requiring both a coefficient of $\geq 0.70$ and all items showing corrected item-total correlation coefficients of $\geq 0.30$ [29].

Convergent and divergent validity of the three CD-RISC versions were assessed by examining Spearman's correlation coefficients between these versions and four convergent reference measures, and five divergent reference measures. Strong correlations $(r \geq 0.50)$ were expected between each of the CD-RISC versions and measures of the related concepts anxiety, depression, passive coping, and life satisfaction. Weak correlations $(r \leq 0.30)$ were expected with cause of SCI, gender, level and completeness of the SCI, and age. We considered validity to be confirmed if, overall, at least $75 \%$ (7:9) of the nine expectations were confirmed [29].

Agreement between the three CD-RISC versions was examined with intraclass correlation coefficients (ICCs). The ICCs and corresponding 95\% confidence intervals (CIs) were calculated based on a single rating, absoluteagreement, with a two-way mixed-effects model. For ICC values, the following interpretation guidelines were used: 'poor' $(<0.50)$, 'moderate' (0.5-0.75), 'good' (0.75-0.90), and 'excellent' reliability $(>0.90)$ [30]. In addition, BlandAltman plots, limits of agreement $\left( \pm 1.96 \times \mathrm{SD}_{\text {difference }}\right)$, and 95\% CIs were provided. For this purpose, total scores on the CD-RISC 10 and CD-RISC 2 were converted to the same 0-100 scale as used in the original version. Incomplete scores on the 25 -item scale were completed by multiplying the mean total score of the questions that were answered by 25 .

\section{Results}

\section{Participant characteristics}

The final sample consisted of 74 individuals with a recently acquired SCI or cauda equina syndrome. Most $(62.2 \%)$
Table 1 Resilience in individuals with SCI in inpatient rehabilitation: internal consistency and outcomes on resilience

\begin{tabular}{|c|c|c|c|}
\hline & CD-RISC 25 & CD-RISC 10 & CD-RISC 2 \\
\hline \multicolumn{4}{|l|}{ Score distribution } \\
\hline \multicolumn{4}{|l|}{ Mean total score (SD) } \\
\hline Raw score & $69.6(13.9)$ & $28.5(6.4)$ & $5.95(1.6)$ \\
\hline Converted score & $69.6(13.9)$ & $71.3(16.1)$ & $74.3(20.0)$ \\
\hline \multicolumn{4}{|c|}{ Lowest and highest total score } \\
\hline Raw score & $32-100$ & $10-40$ & $1-8$ \\
\hline Converted score & $32-100$ & $25-100$ & $13-100$ \\
\hline \multicolumn{4}{|c|}{ Median score (interquartile range) } \\
\hline Raw score & $69.5(63.0-78.3)$ & $29.0(25.0-32.0)$ & $6.0(5.0-7.0)$ \\
\hline Converted score & $69.5(63.0-78.3)$ & $72.5(62.5-80.0)$ & $75.0(62.5-87.5)$ \\
\hline Skewness & -0.38 & -0.60 & -1.12 \\
\hline \multicolumn{4}{|l|}{ Internal consistency } \\
\hline Cronbach's $\alpha$ & 0.90 & 0.86 & 0.66 \\
\hline \multicolumn{4}{|c|}{ Corrected item-total correlation values } \\
\hline$n$ Items $>0.3$ (range) & $22(0.30-0.75)$ & $10(0.42-0.72)$ & $2(0.49)$ \\
\hline$n$ Items $<0.3$ (range) & $3(0.15-0.29)$ & None & None \\
\hline
\end{tabular}

SCI spinal cord injury. Measures: Resilience - Connor-Davidson Resilience Scale (CD-RISC)

were male and the mean age in this group was 55.8 (SD 17.9) years. American Spinal Injury Association (ASIA) Impairment Scale (AIS) grades were: A (13.5\%), B (5.4\%), C (16.2\%), and D (64.9\%). Type of SCI was tetraplegia (44.6\%), paraplegia $(37.8 \%)$, and cauda equina syndrome (17.6\%). Aetiology of SCI was traumatic in $50 \%$ of the participants. In the non-traumatic group, $45.9 \%$ of the SCIs were caused by vascular diseases, benign or malignant tumour, bacterial causes, or neglected hernia symptoms. For three individuals, aetiology was unknown. Mean scores on the HADS were 6.2 (SD 4.5) for anxiety and 6.4 (SD 4.7) for depression. Passive coping, compared with a healthy population, was average with a mean score of 3.2 (SD 1.1). The mean life satisfaction score was 5.0 (SD 2.2).

\section{Outcomes on resilience}

Table 1 shows the scores on the CD-RISC versions. No floor or ceiling effects were found. The negative skewness values indicate that there were less individuals scoring low on the CD-RISCs than that there were individuals scoring high. The scores on the CD-RISC 2 were somewhat higher compared to the other versions and showed a skewness outside the acceptable range.

\section{Internal consistency}

Table 1 also shows that Cronbach's $\alpha$ value was highest (0.90) for the CD-RISC 25 and lowest (0.66) for the CDRISC 2. For the CD-RISC 10 and CD-RISC 2, all questions showed a correlated item-total correlation value of $>0.30$. 
Three of the 74 participants did not answer all $25 \mathrm{CD}$ RISC questions; however, the questions included in the 2item and 10-item versions were completed by all participants.

\section{Concurrent and divergent validity}

The CD-RISC 10 scored most positively on the validity test with $89 \%$ of the expectations confirmed; three out of four convergent validity tests and all five divergent validity tests (Table 2). Overall correlation values on divergent validity were closest to zero on the CD-RISC 10 as well. For the CD-RISC 25 and CD-RISC 2, six out of the nine expectations were confirmed. Depression correlated negatively

Table 2 Convergent and divergent validity

\begin{tabular}{lcll}
\hline Variable & \multicolumn{3}{l}{ Spearman's correlation } \\
\cline { 2 - 4 } & $\begin{array}{l}\text { Resilience } \\
\text { (CD-RISC 25) }\end{array}$ & $\begin{array}{l}\text { Resilience } \\
\text { (CD-RISC 10) }\end{array}$ & $\begin{array}{l}\text { Resilience } \\
\text { (CD-RISC 2) }\end{array}$ \\
\hline Convergent validity & & & \\
Anxiety & $-0.39^{*}$ & $-0.53^{*}$ & $-0.34^{*}$ \\
Depression & $-0.51^{*}$ & $-0.58^{*}$ & $-0.54^{*}$ \\
Passive coping & $-0.46^{*}$ & $-0.51^{*}$ & $-0.30^{*}$ \\
Life satisfaction & 0.14 & 0.22 & 0.18 \\
overall & & & \\
Divergent validity & & -0.03 & -0.20 \\
Cause of SCI & -0.18 & -0.09 & -0.03 \\
Gender & -0.00 & 0.06 & 0.18 \\
Impairment level & 0.03 & & 0.13 \\
(AIS) & & 0.01 & -0.09 \\
Completeness & 0.02 & -0.15 & \\
Age & -0.16 & & \\
\hline
\end{tabular}

Measures: Resilience - Connor-Davidson Resilience Scale (CDRISC), Depression and Anxiety - Hospital Anxiety Depression Scale (HADS), passive coping - Utrechtse Coping List (UCL), Life satisfaction - total score on the two single questions from the Life Satisfaction questions (2LS), Impairment - American Spinal Injury Association (ASIA) Impairment Scale. ${ }^{*} p<0.01$ with resilience according to all three CD-RISC scales and life satisfaction did not correlate with resilience according to each of them.

\section{Evaluation of agreement}

Table 3 shows that the CD-RISC 25 and CD-RISC 10 model accounts for the highest explained variance $(81.5 \%)$ as confirmed by the correlation between those scales. The degree of this correlation (ICC $=0.90)$ and agreement between them (good to excellent) showed high reliability. Bland-Altman plots with limits of agreement and 95\% CIs are displayed in Fig. 1. All plots showed that the differences between the CD-RISC versions are higher in individuals who scored lower on resilience.

\section{Discussion}

The purpose of the present study was to compare the psychometric characteristics of the CD-RISC with 25,10 , and 2 items and to assess the agreement between these versions among individuals with a recently acquired SCI during inpatient rehabilitation. Total CD-RISC scores were only skewed on the CD-RISC 2. No floor and ceiling effects were found and internal consistency of the 25-, 10-, and 2item scales was good to moderate. Good convergent validity was shown only for the CD-RISC 10 and agreement was highest between the CD-RISC 25 and CD-RISC 10 model. Internal consistency statistics of the three CD-RISCs were most satisfying for the CD-RISC 10 . Further, only 3 out of the 25 items of the CD-RISC 25 had a corrected itemtotal correlation value above the threshold of 0.3 , while for the CD-RISC 10 this value accounted for all 10 items. However, since Cronbach's alpha is sensitive to the number of items in a scale and the CD-RISC 2 has only two questions, this shortest version might be seen as internally consistent as well. Our results are comparable with the Cronbach $\alpha$ values $(0.93$ on the CD-RISC $25,0.88$ on the

Table 3 Agreement measures

\begin{tabular}{|c|c|c|c|c|c|}
\hline Model & $\begin{array}{l}\text { Correlation } \\
\text { coefficient }\end{array}$ & $\begin{array}{l}\text { Explained } \\
\text { variance }(\%)\end{array}$ & $\begin{array}{l}\text { Intraclass correlation } \\
\text { coefficient (ICC) } \\
\text { (single measures) }\end{array}$ & $\begin{array}{l}\text { ICC 95\% } \\
\text { confidence Interval } \\
\text { (lower bound to } \\
\text { upper bound) }\end{array}$ & Agreement \\
\hline $\begin{array}{l}\text { CD-RISC } 25 \text { and } \\
\text { CD-RISC } 10\end{array}$ & 0.90 & 81.5 & 0.90 & 0.85 to 0.94 & Good to excellent \\
\hline $\begin{array}{l}\text { CD-RISC } 25 \text { and } \\
\text { CD-RISC } 2\end{array}$ & 0.55 & 30.3 & 0.51 & 0.32 to 0.66 & Poor to moderate \\
\hline $\begin{array}{l}\text { CD-RISC } 10 \text { and } \\
\text { CD-RISC } 2\end{array}$ & 0.70 & 48.6 & 0.69 & 0.55 to 0.79 & Moderate to good \\
\hline
\end{tabular}

Measures: Resilience - Connor-Davidson Resilience Scale (CD-RISC). All three models were statistically significant 


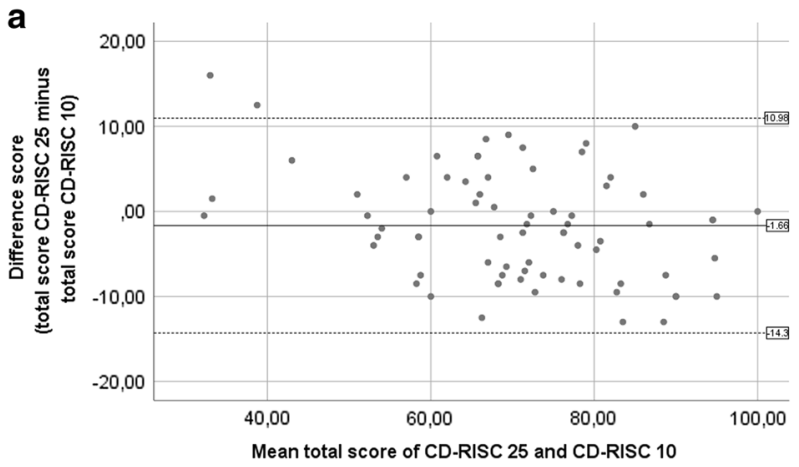

b
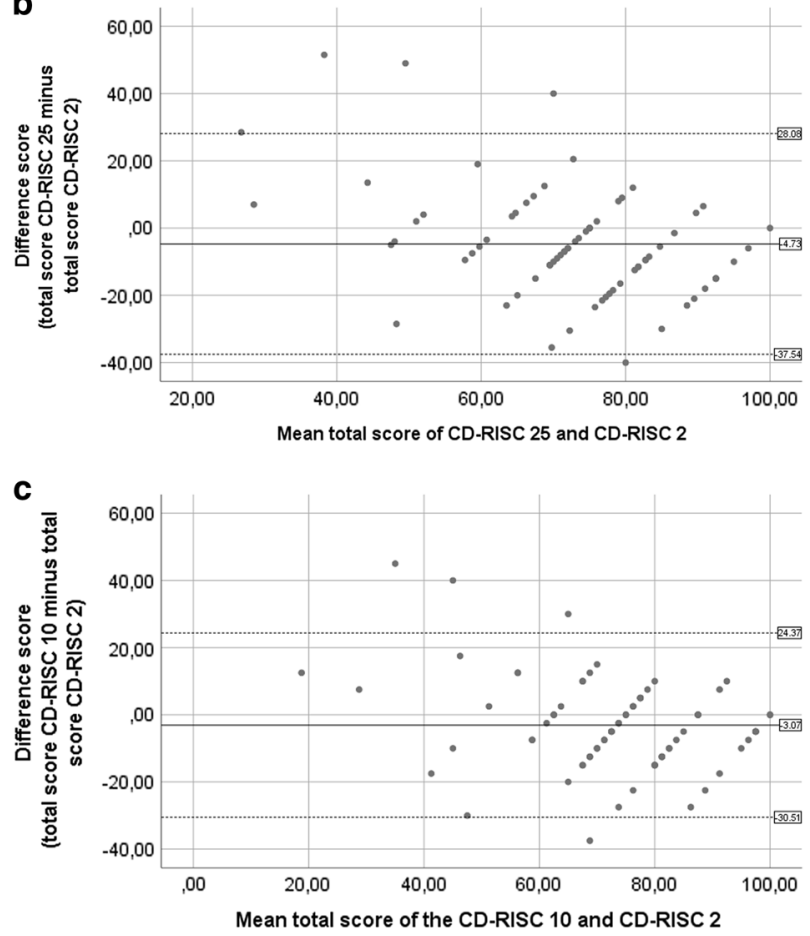

Fig. 1 Bland-Altman plots of the differences between total scores on the Connor-Davidson Resilience Scale (CD-RISC)s compared related to the mean total scores on these CD-RISCs. Each dot represents an individual participant with $\mathrm{SCI}$ in a rehabilitation setting $(\mathrm{N}=74)$. a Differences between total scores on the 25-item and the 10-item CDRISCs related to the mean total scores on the 25 -item and the 10 -item CD-RISC. The mean difference score $(-1.7)$ and limits of agreement are represented by the horizontal lines. The $95 \%$ CI of the difference was -3.15 to -0.16 . b Differences between total scores on the 25 item and the 2-item CD-RISCs related to the mean total scores on the 25 -item and the 2-item CD-RISC. The mean difference score $(-4.7)$ and limits of agreement are represented by the horizontal lines. The $95 \% \mathrm{CI}$ of the difference was -8.61 to -0.85 . c Differences between total scores on the 10-item and the 2-item CD-RISCs related to the mean total scores on the 10 -item and the 2 -item CD-RISC. The mean difference score $(-3.1)$ and limits of agreement are represented by the horizontal lines. The $95 \% \mathrm{CI}$ of the difference was -6.35 to 0.20

CD-RISC 10, and 0.76 on the CD-RISC 2) found among individuals with severe accidental injuries [9].

It is not surprising that all three scales showed a correlation between resilience and depression; several studies have reported this correlation $[12,15]$. However, it is surprising that the original scale and the CD-RISC 2 did not correlate $\geq 75 \%$ with the convergent criteria, since many studies reported correlations with anxiety, passive coping, and life satisfaction $[12,14,18,20]$. These correlation differences between the CD-RISC 10, and the 25- and 2-item versions might partly be explained by the setting of our study in which the individuals completed the screening early after the onset of the SCI, in contrast with measurement time periods (post rehabilitation) in other studies $[18,20]$. However, the other two studies both collected data at three time periods, including shortly after admission. At that time period, resilience was correlated to anxiety and satisfaction with life as well $[12,14]$. Another explanation might be found in the diverging instruments used to assess the criteria measurements. In one of the studies, anxiety was measured by the Depression, Anxiety, and Stress Scale 21 [18]. Satisfaction with life was examined with the Satisfaction With Life Scale in another study [14]. At the start of the present study, it was considered to test correlations between resilience with physical functioning, mobility, pain, and fatigue as well. However, we did not find studies that repeatedly confirmed such correlations and, therefore, these measures were not included in the validity tests.

According to the agreement statistics, the questions from CD-RISC 10 represent the original CD-RISC 25 the best. High agreement was expected since 10 items are common to both scales and those 10 items were chosen based on multiple exploratory and confirmatory factor analyses [8]. The lower agreement between the CD-RISC 2 and the CDRISC 25 was not surprisingly as well, since only two items are common to both scales. This effect is increased by the fact that both CD-RISC 2 questions are classifiable as 'hardiness' and were chosen based only on subjective reasons and not on empirical criteria [9]. Taking in mind the time that could be saved and the moderate agreement data between the CD-RISC 2 and CD-RISC 10, one still may consider the clinical use of the CD-RISC 2 as well. On the contrary, one may also suggest that the CD-RISC 2 comprises a one-sided approach of the broad concept of resilience. This idea is in accordance with the difference between the explained variance of the CD-RISC 10 and CD-RISC 2 with the overall scale $(81.5-30.3=51.2 \%)$.

Most of the individuals with SCI in a rehabilitation setting showed high resilience according to the CD-RISC results. This finding is comparable with a study in which was found that $54 \%$ of the 80 individor multiple trauma were resili/or multiple trauma were resilient [31].

\section{Limitations of the current study}

A number of limitations need to be considered. First, the study was not subjected to a sample size calculation. 
Nonetheless, data from 74 individuals were expected to be sufficient for this type of study [29]. Second, with respect to representativeness, the mean age in this group was higher and the percentage of individuals with a complete injury was lower in comparison with individuals from similar populations [11, 13, 15]. Third, participants were not asked about their history of other adversities. Experience with previous adversities may have influenced the way individuals cope with SCI. Likewise, it may also have influenced their ideas about their coping with such adversities, and therefore how they answered the CD-RISC questions. Another form of response bias might be caused by the selfreporting design of the instruments. Fourth, participants completed the CD-RISC 25 only; the results of the CDRISC 10 and CD-RISC 2 were derived from this original instrument. Hence, the actual answers on a shorter version might have been slightly different from the answers on the original version since these answers might be influenced by the presence of other questions. A final limitation of the current study is that only Dutch individuals participated. This means that generalisation of the results to other countries asks for caution.

\section{Conclusions}

Our study found good to excellent psychometric properties of the CD-RISC 10, confirming its reliability and validity in individuals with SCI in inpatient rehabilitation. The CDRISC 2 showed moderate psychometric properties and might be used if time is of the essence. Nonetheless, this 2item scale yields a less complete impression of someone's resilience. Future research should for example evaluate whether low levels of resilience during the first weeks of rehabilitation predict lower psychological functioning at the end of inpatient rehabilitation. Additional research may focus on the practical use of the CD-RISC 10 in individuals with a recently acquired SCI in a rehabilitation setting. Information from such studies could serve as guidance for clinical, positive psychology interventions for individuals with a recently acquired SCI in a rehabilitation setting.

\section{Data archiving}

The datasets analysed during the current study are available from the corresponding author on reasonable request.

Acknowledgements The authors would like to thank the participating research assistants in Rehabilitation Centre De Hoogstraat.

Author contributions HK was responsible for conceiving and designing the study, the data analysis, and drafting the paper. CCMvL was responsible for conceiving and designing the study, the data collection and providing feedback on the paper. JMS-S was responsible for conceiving and designing the study, and providing feedback on the paper. MWMP was responsible for conceiving and designing the study, and providing feedback on the paper.

Funding This project was funded by a grant from the Dutch Health Care Institute (registration number 2017033112) through the Dutch Spinal Cord Injury Association (registration number SAB049).

\section{Compliance with ethical standards}

Conflict of interest The authors declare that they have no conflict of interest.

Statement of ethics We certify that all applicable institutional and governmental regulations concerning the ethical use of human volunteers were followed during the course of this research.

Publisher's note: Springer Nature remains neutral with regard to jurisdictional claims in published maps and institutional affiliations.

\section{References}

1. Connor KM, Davidson JRT. Development of a new resilience scale: The Connor-Davidson Resilience scale (CD-RISC). Depress Anxiety. 2003;18:76-82.

2. Martz E, Livneh H, Priebe M, Wuermser LA, Ottomanelli L. Predictors of psychosocial adaptation among people with spinal cord injury or disorder. Arch Phys Med Rehabil. 2005; 86:1182-92.

3. Van Leeuwen CMC, Kraaijeveld S, Lindeman E, Post MWM. Associations between psychological factors and quality of life ratings in persons with spinal cord injury: a systematic review. Spinal Cord. 2012;50:174-87.

4. Russell HF, Richardson EJ, Bombardier CH, Dixon TM, Huston TA, Rose J, et al. Professional standards of practice for psychologists, social workers, and counselors in SCI rehabilitation. J Spinal Cord Med. 2016;39:127-45.

5. Dunn DS, Dougherty SB. Prospects for a positive psychology of rehabilitation. Rehabil Psychol. 2005;50:305-11.

6. Joyce S, Shand F, Tighe J, Laurent SJ, Bryant RA, Harvey SB. Road to resilience: a systematic review and meta-analysis of resilience training programmes and interventions. BMJ Open. 2018;8:e017858.

7. Windle G, Bennett KM, Noyes J. A methodological review of resilience measurement scales. Health Qual Life Outcomes. 2011;9:8.

8. Campbell-Sills L, Stein MB. Psychometric analysis and refinement of the Connor-Davidson Resilience Scale (CD-RISC): validation of a 10-item measure of resilience. J Trauma Stress. 2007;20:1019-28.

9. Li P, Jiaja Z, Han C, Yu Z, Min L, Yongju Y, et al. Comparison among different versions of Connor-Davidson Resilience Scale (CD-RISC) in rehabilitation patients after unintentional injury. $\mathrm{J}$ Psychiatry. 2014;17:1-5.

10. Catalano D, Chan F, Wilson L, Chiu CY, Muller VR. The buffering effect of resilience on depression among individuals with spinal cord injury: a structural equation model. Rehabil Psychol. 2011;56:200-11.

11. Guest RR, Craig A, Nicholson Perry K, Tran Y, Ephraums C, Hales A, et al. Resilience following spinal cord injury: a prospective controlled study investigating the influence of the provision of group cognitive behavior therapy during inpatient rehabilitation. Rehabil Psychol. 2015;60:311-21. 
12. Shin J-I, Chae J-H, Min J-A, Lee C-U, Hwang S-I, Lee B-S, et al. Resilience as a possible predictor for psychological distress in chronic spinal cord injured patients living in the community. Ann Rehabil Med. 2012;36:815.

13. White B, Driver S, Warren AM. Resilience and indicators of adjustment during rehabilitation from a spinal cord injury. Rehabil Psychol. 2010;55:23-32.

14. Edwards KA, Alschuler KA, Ehde DM, Battalio SL, Jensen MP. Changes in resilience predict function in adults with physical disabilities: a longitudinal study. Arch Phys Med Rehabil. 2017;98:329-36.

15. Dodd Z, Driver S, Warren A, Riggs S, Clark M. Effects of adult romantic attachment and social support on resilience and depression in individuals with spinal cord injuries. Top Spinal Cord Inj Rehabil. 2015;21:156-65.

16. Driver S, Warren AM, Reynolds M, Agtarap S, Hamilton R, Trost $\mathrm{Z}$, et al. Identifying predictors of resilience at inpatient and 3month post-spinal cord injury. J Spinal Cord Med. 2016;39:7784 .

17. Kilic SA, Dorstyn DS, Guiver NG. Examining factors that contribute to the process of resilience following spinal cord injury. Spinal Cord. 2013;51:553-7.

18. Silverman AM, Molton IR, Alschuler KN, Ehde DM, Jensen MP. Resilience predicts functional outcomes in people aging with disability: a longitudinal investigation. Arch Phys Med Rehabil. 2015;96:1262-8.

19. Terrill AL, Molton IR, Ehde DM, Amtmann D, Bombardier CH, Smith $\mathrm{AE}$, et al. Resilience, age, and perceived symptoms in persons with long-term physical disabilities. J Health Psychol. 2016;21:640-9.

20. Vaishnavi S, Connor K, Davidson JRT. An abbreviated version of the Connor-Davidson Resilience Scale (CD-RISC), the CDRISC2: psychometric properties and applications in psychopharmacological trials. Psychiatry Res. 2007;152:293-7.

21. Zigmond AS, Snaith RP. The hospital anxiety and depression scale. Acta Psychiatr Scandunavica. 1983;67:361-70.
22. Kennedy P, Lude P, Elfström ML, Smithson E. Cognitive appraisals, coping and quality of life outcomes: a multi-centre study of spinal cord injury rehabilitation. Spinal Cord. 2010;48:762-9.

23. Woolrich RA, Kennedy P, Tasiemski T. A preliminary psychometric evaluation of the Hospital Anxiety and Depression Scale (HADS) in 963 people living with a spinal cord injury. Psychol Health Med. 2006;11:80-90.

24. Sakakibara BM, Miller WC, Orenczuk SG, Wolfe DL. A systematic review of depression and anxiety measures used with individuals with spinal cord injury. Spinal Cord. 2009;47:841-51.

25. Schreurs P, van de Willige G, JF B, B T, Graus G. De Utrechtse Coping Lijst: UCL. Amsterdam: Pearson; 1993.

26. van Diemen T, Scholten EW, van Nes IJ, Geertzen JH, Post MW. Self-management and self-efficacy in patients with acute spinal cord injuries: protocol for a longitudinal cohort study. JMIR Res Protoc. 2018;7:e68.

27. Voerman GE, Erren-Wolters CV, Fleuren JF, Hermens HJ, Geurts AC. Perceived spasticity in chronic spinal cord injured patients: associations with psychological factors. Disabil Rehabil. 2010;32:775-80.

28. Post MW, Van Leeuwen CM, Van Koppenhagen CF, De Groot S. Validity of the life satisfaction questions, the life satisfaction questionnaire, and the satisfaction with life scale in persons with spinal cord injury. Arch Phys Med Rehabil. 2012;93: $1832-7$.

29. Terwee CB, Bot SDM, de Boer MR, van der Windt DAWM, Knol DL, Dekker J, et al. Quality criteria were proposed for measurement properties of health status questionnaires. J Clin Epidemiol. 2007;60:34-42.

30. Koo TK, Li MY. A guideline of selecting and reporting intraclass correlation coefficients for reliability research. J Chiropr Med. 2016;15:155-63.

31. Quale AJ, Schanke A. Resilience in the face of coping with a severe physical injury: a study of trajectories of adjustment in a rehabilitation setting. Rehabil Psychol. 2010;55:12-22. 